\title{
OBSERVATION OF THE IMPULSIVE PHASE OF A SIMPLE FLARE
}

\author{
E. TANDBERG-HANSSEN, P. KAUFMANN, ${ }^{1}$ E. J. REICHMANN, D. L. \\ TEUBER, R. L. MOORE, L. E. ORWIG, ${ }^{2}$ and H. ZIRIN ${ }^{3}$
}

NASA, Marshall Space Flight Center, AL 35812, U.S.A.

(Received 5 September, 1983)

\begin{abstract}
We present a broad range of complementary observations of the onset and impulsive phase of a fairly large (1B, M1.2) but simple two-ribbon flare. The observations consist of hard X-ray flux measured by the SMM HXRBS, high-sensitivity measurements of microwave flux at $22 \mathrm{GHz}$ from Itapetinga Radio Observatory, sequences of spectroheliograms in UV emission lines from $\mathrm{OV}\left(T \approx 2 \times 10^{5} \mathrm{~K}\right)$ and $\mathrm{FexxI}$ $\left(T \approx 1 \times 10^{7} \mathrm{~K}\right.$ ) from the SMM UVSP, $\mathrm{H} \alpha$ and $\mathrm{He} \mathrm{I} \mathrm{D}_{3}$ cine-filtergrams from Big Bear Solar Observatory, and a magnetogram of the flare region from the MSFC Solar Observatory. From these data we conclude:

(1) The overall magnetic field configuration in which the flare occurred was a fairly simple, closed arch containing nonpotential substructure.

(2) The flare occurred spontaneously within the arch; it was not triggered by emerging magnetic flux.

(3) The impulsive energy release occurred in two major spikes. The second spike took place within the flare arch heated in the first spike, but was concentrated on a different subset of field lines. The ratio of Ov emission to hard X-ray emission decreased by at least a factor of 2 from the first spike to the second, probably because the plasma density in the flare arch had increased by chromospheric evaporation.

(4) The impulsive energy release most likely occurred in the upper part of the arch; it had three immediate products:

(a) An increase in the plasma pressure throughout the flare arch of at least a factor of 10 . This is required because the FexxI emission was confined to the feet of the flare arch for at least the first minute of the impulsive phase.

(b) Nonthermal energetic ( $\sim 25 \mathrm{keV})$ electrons which impacted the feet of the arch to produce the hard $\mathrm{X}$-ray burst and impulsive brightening in $\mathrm{OV}$ and $\mathrm{D}_{3}$. The evidence for this is the simultaneity, within $\pm 2 \mathrm{~s}$, of the peak OV and hard X-ray emissions.

(c) Another population of high-energy $(\sim 100 \mathrm{keV})$ electrons (decoupled from the population that produced the hard X-rays) that produced the impulsive microwave emission at $22 \mathrm{GHz}$. This conclusion is drawn because the microwave peak was $6 \pm 3 \mathrm{~s}$ later than the hard X-ray peak.
\end{abstract}

\section{Introduction}

On November 1, 1980, active region Boulder No. AR 2776 had moved well on to the disk when a flare occurred around 19:15 UT in the northern part of the region (coordinates N17, E64). AR 2776 had been watched closely both by the Solar Maximum Mission (SMM) spaceborne instruments and by numerous ground-based observatories. Very good data were obtained of a variety of flare manifestations; e.g., radio and hard $\mathrm{X}$-ray burst, soft X-ray continuum, and UV line emissions, as well as $\mathrm{H} \alpha$ and $\mathrm{He} \mathrm{I}_{3}$ line pictures, and preflare magnetic field maps. In particular, the onset and flash phase of this $1 \mathrm{~B}, \mathrm{M} 1.2$ flare is documented, and it is the purpose of this paper to analyze and

${ }^{1}$ INPE, Instituto de Pesquisas Espaciais, 12200 S. José dos Campos, SP Brazil.

2 NASA, Goddard Space Flight Center, MD 20771, U.S.A.

${ }^{3}$ Big Bear Solar Observatory, Caltech, Pasadena, CA 91125, U.S.A. 
discuss this broad SMM and ground-based data base, with the goal of offering a physical picture of the initial phases of the flare.

In Section 2, we present the different data used in this study. The flare onset and morphological studies will be discussed in Section 3, while Section 4 treats details of the time development of the impulsive emissions. A general discussion of the flare is presented in Section 5.

\section{Observations}

The observations of the November 1, 1980, flare demonstrate the importance of obtaining correlated, simultaneous ground-based and spaceborne data on fast changing solar activity manifestations like flares. The relevant data on this flare are presented in Table I and in the figures. It is very fortunate that a wide variety of instruments, sampling the electromagnetic spectrum from radiowaves to hard $\mathrm{X}$-rays, caught the very beginning of the flare and could follow the emissions through the impulsive phase and well into the thermal phase and decay.

TABLE I

The data set for our flare

\begin{tabular}{llll}
\hline Type & $\begin{array}{l}\text { Spectral region, } \\
\text { frequency or energy }\end{array}$ & $\begin{array}{l}\text { Time } \\
\text { resolution }\end{array}$ & Source \\
\hline Radio & $22 \mathrm{GHz}$ & $1 \mathrm{~ms}$ & Itapetinga \\
Optical & $\mathrm{HI}, 6563 \AA(\mathrm{H} \alpha)$ & $15 \mathrm{~s}$ & BBSO \\
& FeI, $5250 \AA$ polarimeter & & MSFC \\
& HeI, $5876 \AA\left(\mathrm{D}_{3}\right)$ & $15 \mathrm{~s}$ & BBSO \\
UV & Ov, $1371 \AA$ & $1.2 \mathrm{~s}, 25 \mathrm{~s}$ & SMM/UVSP \\
& FeXXI, $1354 \AA$ & $1.2 \mathrm{~s}, 25 \mathrm{~s}$ & SMM/UVSP \\
Soft X-rays & $1-8 \AA(\mathrm{XL})$ & $3 \mathrm{~s}$ & NOAA/GOES \\
& $0.5-4 \AA(\mathrm{XS})$ & $3 \mathrm{~s}$ & NOAA/GOES \\
Hard X-rays & $28-100 \mathrm{keV}$ & $0.128 \mathrm{~s}$ & SMM/HXRBS \\
\hline
\end{tabular}

The radio emissions at $22 \mathrm{GHz}$ from the flaring plasma were recorded with the $13.7 \mathrm{~m}$ dish at the Itapetinga Radio Observatory of the Instituto de Pesquisas Espaciais in Brazil beginning during the impulsive phase, but catching the main spike (see Figure 1b). The equipment has a very high sensitivity and high temporal resolution $(1 \mathrm{~ms})$ (Kaufmann et al., 1982) which permits the observation of the fluctuations discussed in Section 4.

In $\mathrm{H} \alpha$ the event as observed at the Big Bear Solar Observatory (BBSO) was reported to start at 19:15 UT and reach maximum as a 1B flare at 19:22 UT. The flare consisted of two distinct separated brightenings. These two $\mathrm{H} \alpha$ ribbons at $19: 22 \mathrm{UT}$ are used as a position reference for the magnetogram and UV images in Figure 2. He I $D_{3}(5876 \AA)$ pictures of the flare were also obtained at BBSO, and the evolution of the two parts of 


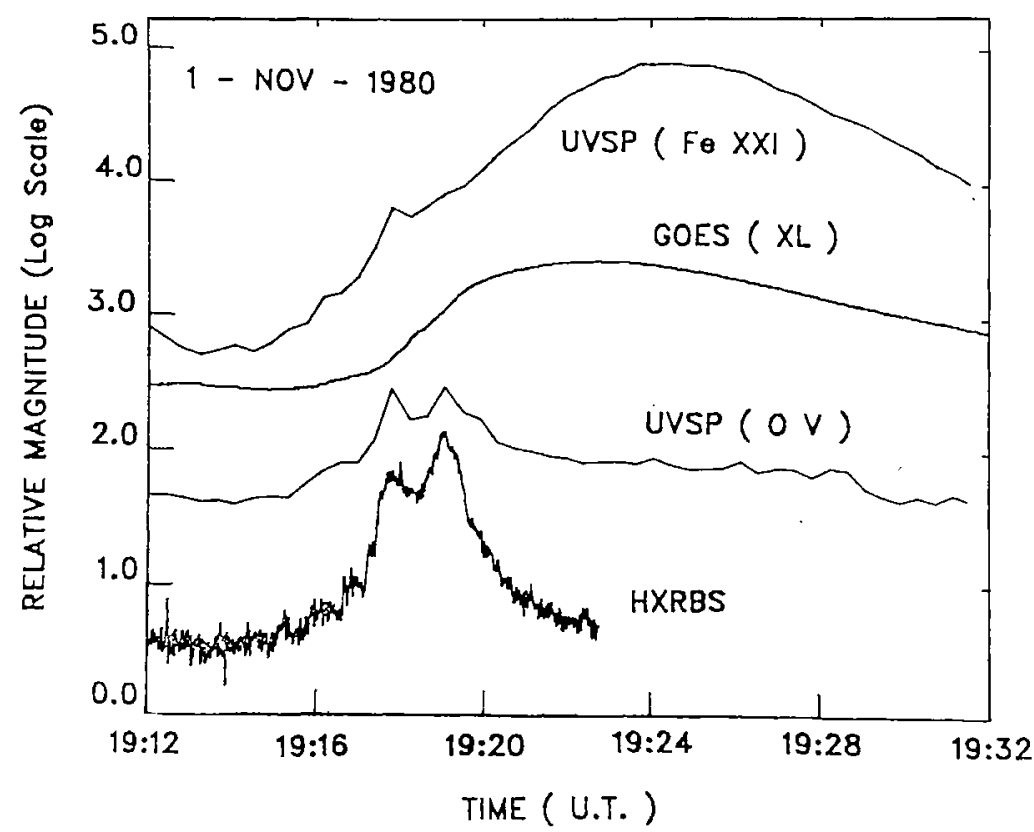

Fig. 1a. Comparison of the time profile of the soft X-ray (1-8 $\AA$ ) emission with the time profiles of the hard $\mathrm{X}$-ray (photon energy $\geq 28 \mathrm{keV}$ ) emission, the emission in the $1371 \AA$ line of $\mathrm{OV}\left(T \approx 2 \times 10^{5} \mathrm{~K}\right)$, and the emission in the $1354 \AA$ line of $\mathrm{FeXXI}\left(T \approx 1 \times 10^{7} \mathrm{~K}\right)$, from before flare onset, through the impulsive phase, until well after the maximum of the gradual phase. The soft X-ray and hard X-ray profiles are plotted with the full time resolution of the GOES ( $3 \mathrm{~s})$ and HXRBS $(0.128 \mathrm{~s})$ instruments. The Ov and Fe XXI profiles are for the emission observed from that portion of the northern flare foot that fell within the $3 \times 3$ pixel raster, and are plotted with uniform time resolution of $25 \mathrm{~s}$ (see text). The OV profile is markedly more similar to the hard X-ray profile than to the soft X-ray profile, whereas the FexXI profile is more similar to the soft X-ray profile.

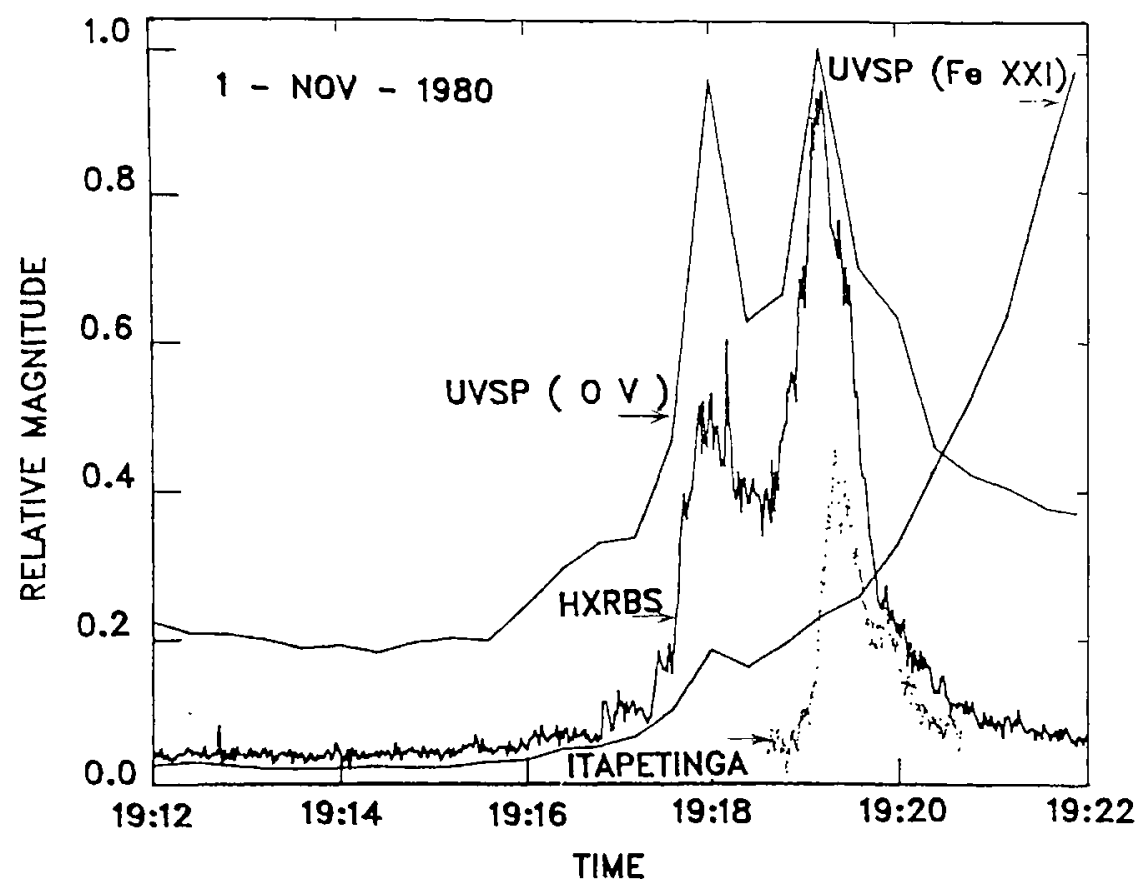

Fig. 1b. Comparison of the time profile of the $22 \mathrm{GHz}$ emission with the hard X-ray, OV, and Fe XxI time profiles through the impulsive phase. The $22 \mathrm{GHz}$ observations began between the two main spikes of the impulsive phase. The time resolutions of the hard X-ray, OV, and Fe XXI plots are the same as in Figure 1a; the $22 \mathrm{GHz}$ data are plotted with a time resolution of $0.4 \mathrm{~s}$. The ratio of $\mathrm{OV}$ emission to hard X-ray emission at the peak of the second spike is about a factor of 2 less than at the peak of the first spike. 


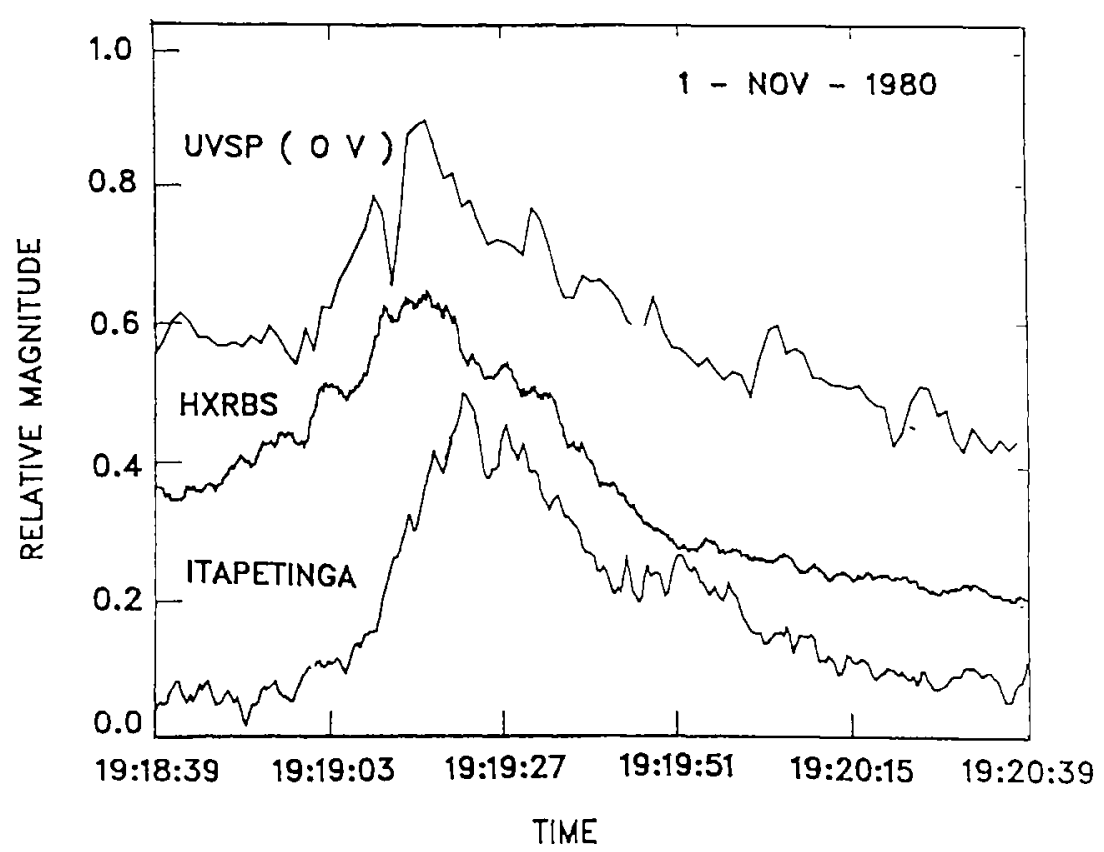

Fig. 1c. Comparison of the $\mathrm{Ov}$, hard X-ray, and $22 \mathrm{GHz}$ time profiles in the second spike of the impulsive phase. Here, the $\mathrm{OV}$ data are plotted with their full time resolution of $1.2 \mathrm{~s}$; the hard X-ray plot has $0.128 \mathrm{~s}$ resolution; the $22 \mathrm{GHz}$ plot has $0.4 \mathrm{~s}$ resolution. The $22 \mathrm{GHz}$ peak is seen to lag the $\mathrm{OV}$ and hard X-ray peaks by several seconds.

MSFC-RTSM LONGITUDINAL FIELD

1-NOV $1980 \quad$ 19:02 U.T.

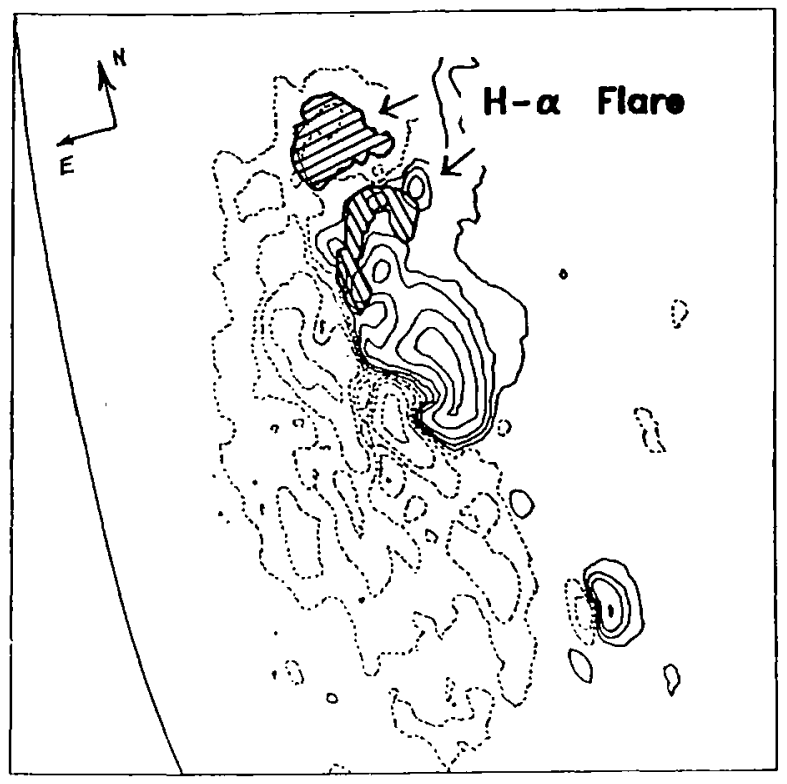

Fig. 2a. Magnetogram of $\mathrm{AR} 2776$ with $\mathrm{H} \alpha$ ribbons at flare maximum superposed. The heliographic direction of the segment of the polarity inversion line straddled by the flare is nearly east-west. 
the flare can be studied with good time resolution. The two separate parts of the flare occurred in regions of opposite magnetic polarity as determined from the polarization of the Fe I 5250 A line by the NASA/MSFC magnetograph (Hagyard et al., 1982); see Figure 2a which shows a magnetogram of AR 2776. We shall refer to the locations of these two distinct brightenings as the two foot-points, a northern and a southern.

The Ultraviolet Spectrometer and Polarimeter (UVSP, see Woodgate et al., 1980), on SMM continuously made spectroheliograms of the flare region from before the flare to well into the decay phase. These intensity maps were obtained in simultaneous pairs, one in the Ov, 1371 A line, the other in the FexXI, $1354 \AA$ Aine. Each spectroheliogram
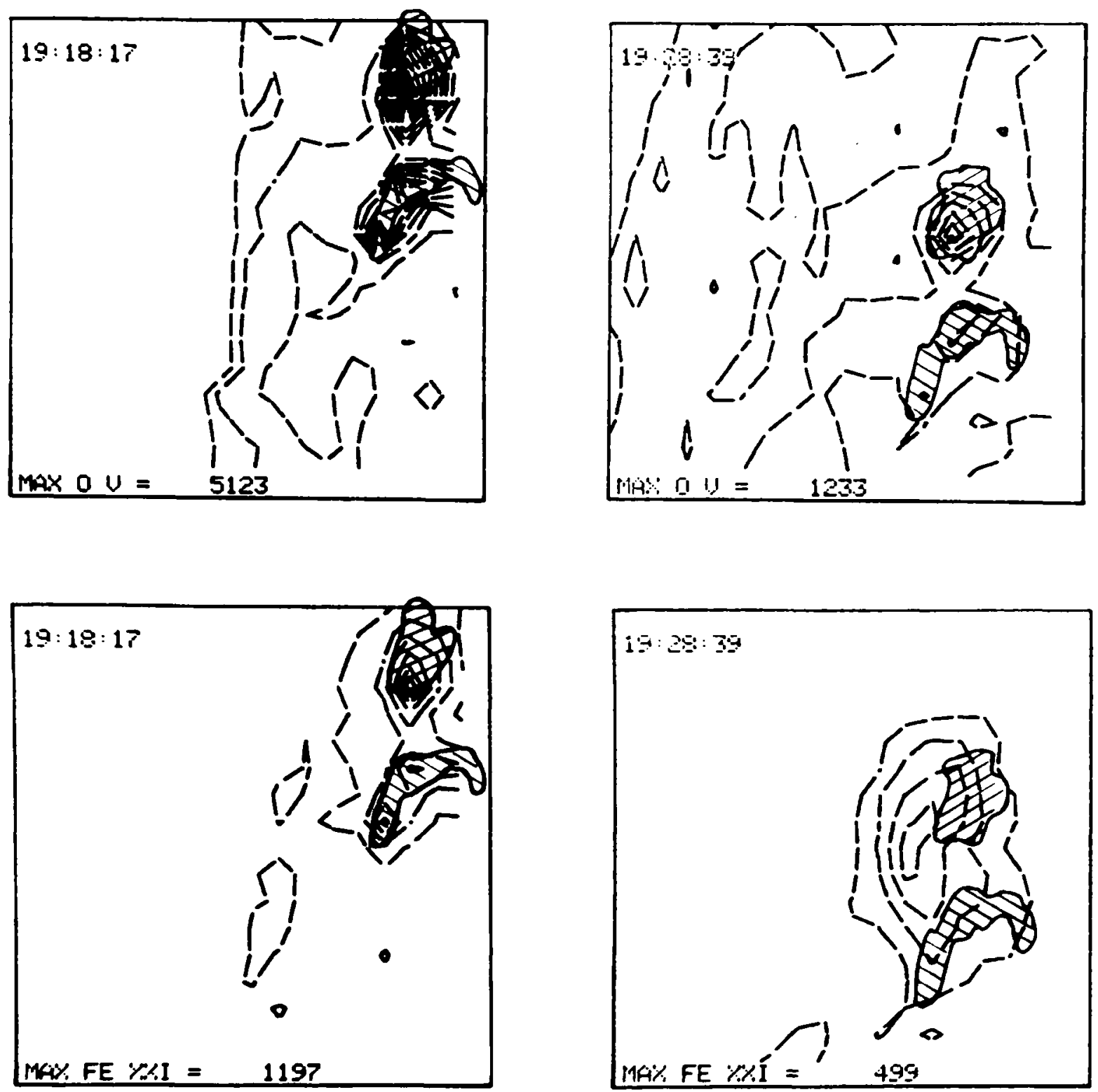

Fig. 2b. Ov (top) and Fexxr (bottom) emission patterns in the impulsive phase (left) and in the gradual phase (right) with the $\mathrm{H} \alpha$ ribbons superposed. In the impulsive phase, the $\mathrm{O} v$ and Fe XXI emissions are both concentrated in the area of the $\mathrm{H} \alpha$ ribbons. In the gradual phase, the $\mathrm{OV}$ emission is still concentrated in the feet, but the Fe XXI emission is centered over the inversion line. In all UV spectroheliograms in this paper, solar north is up and east is to the left. 

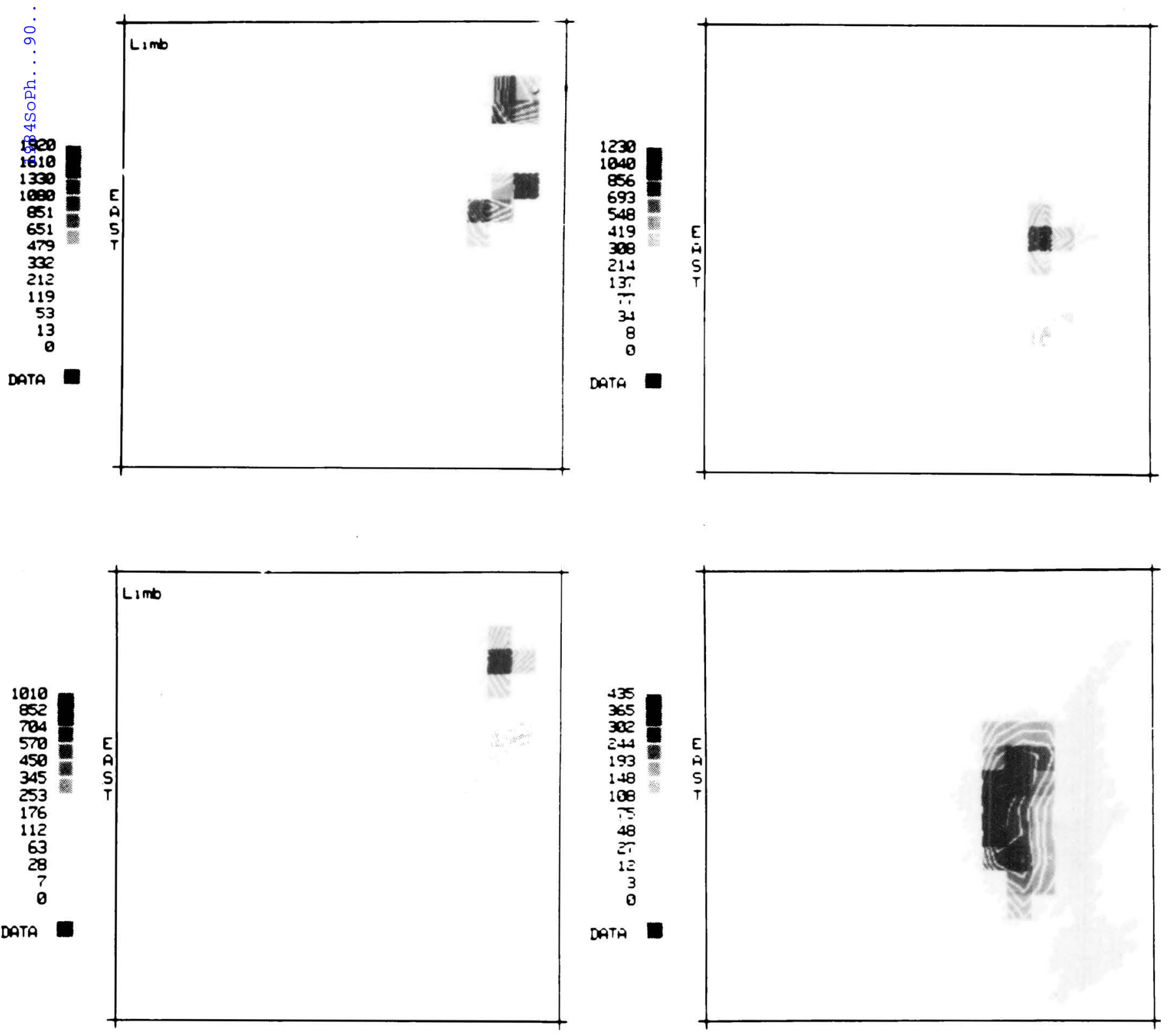

Fig. 2c. Same as Figure $2 b$, but showing the brighter pixels from which the contours in Figure $2 b$ were derived. This emphasizes the concentration of the Ov emission in the flare feet both during and after the impulsive phase, the concentration of the FeXXI emission in the feet in the impulsive phase, and the arch form of the FeXXI emission in the gradual phase.

of this set is a raster of 10 arc sec square pixels. From before the flare until half way through the impulsive phase, 3 arc min square rasters (18 pixels across) were taken at a rate of one pair per $25 \mathrm{~s}$. These rasters covered most of the flare area (Figure 3). Then, to increase the time resolution to $1.2 \mathrm{~s}$, the raster size was reduced to $30 \mathrm{arc} \mathrm{sec} \mathrm{square}$ ( 3 pixels across) for the rest of the impulsive phase and through the maximum of the thermal flare. This smaller raster covered only the eastern part of the northern foot of the flare (Figure 3a). Several minutes after flare maximum, the raster size was increased back to 3 arc min. The time plots of $\mathrm{OV}$ and FeXXI emission in Figure 1 are for the emission from the 30 arc sec square area observed throughout the flare. 


\section{AR 27761 - NOV - 1980 Fe XXI}

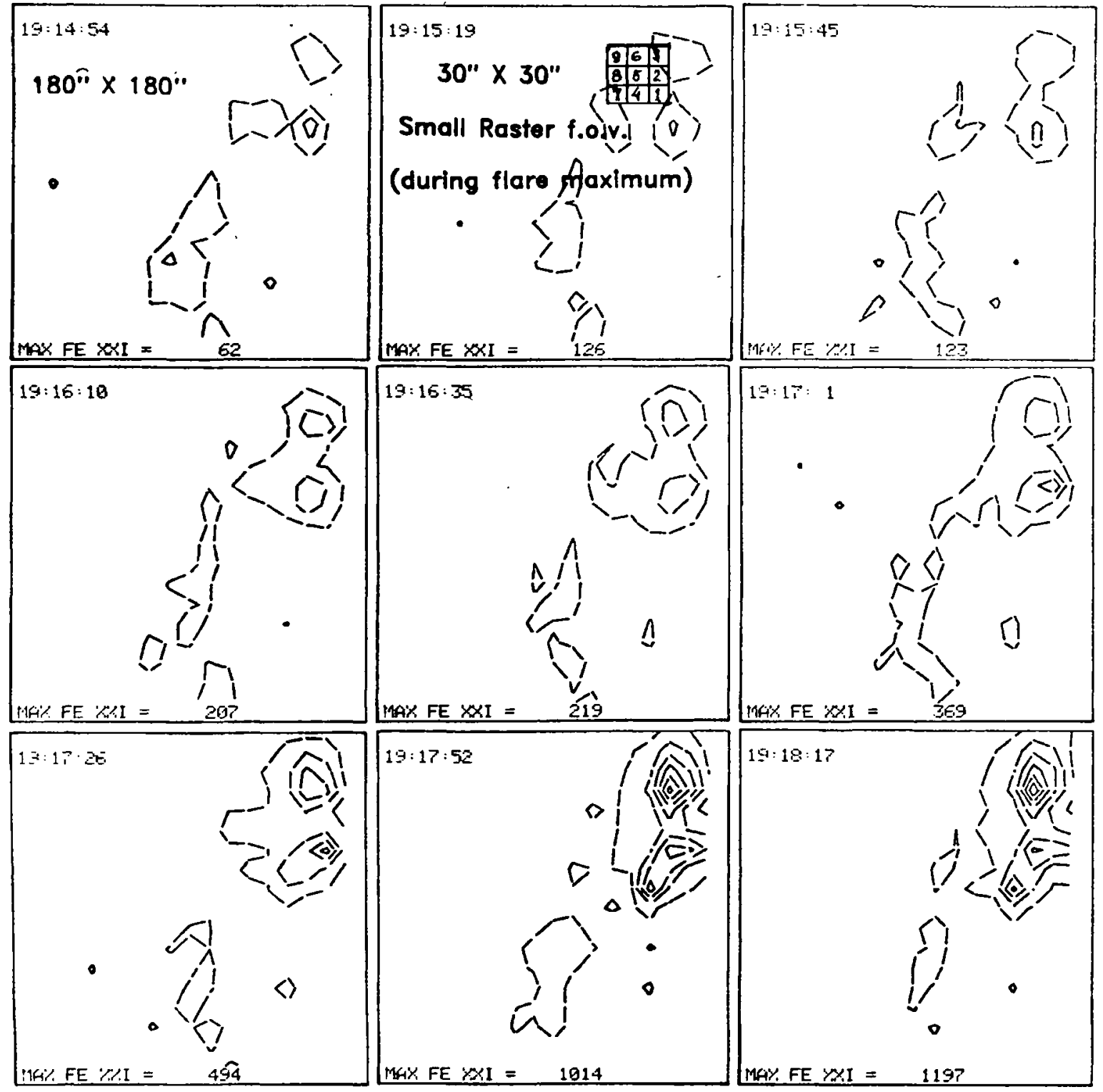

Fig. 3a. FexxI spectroheliograms in the onset and impulsive phase. The time between frames is $25 \mathrm{~s}$. The position of the small raster that was scanned every $1.2 \mathrm{~s}$ from $19: 18: 30$ to $19: 28: 39$ UT is shown in the second frame. The first frame is the first spectroheliogram in which the flare onset in Fe XXI was detectable. The bottom row shows that the FexXI emission was concentrated in the flare feet during at least the first minute of the impulsive phase.

In soft X-rays $(0.5-4 \AA$ and $1-8 \AA$ channels, obtained with the NOAA GOES satellite), the flare reached importance M1.2 (see Figure 1). Finally, the Hard X-Ray Burst Spectrometer (HXRBS) (Orwig et al., 1980) on the SMM observed the flare in its energy channels between 28 and $100 \mathrm{keV}$; see Figure 1. 


\section{AR $27761-\mathrm{NOV}-19800 \mathrm{~V}$}
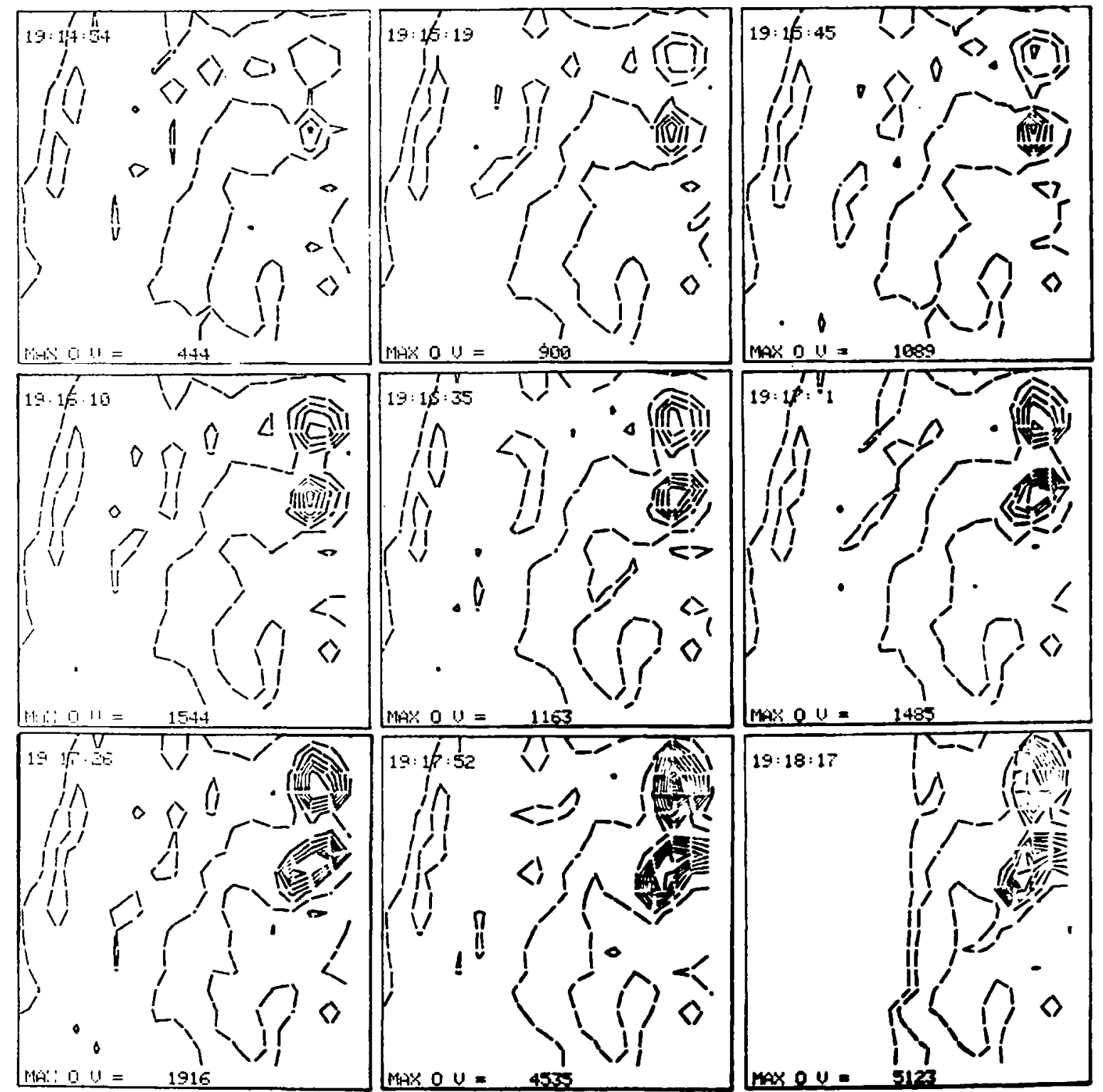

Fig. 3b. Ov spectroheliograms obtained simultaneously with the FexxI spectroheliograms in Figure 3a. The limb is seen near the east edge of each frame but the last; only the west half of the last frame was scanned before switching to the small raster. The first frame is the first spectroheliogram in which the flare onset in OV was detectable.

\section{Temporal and Morphological Development}

Both in the soft X-ray flux observed by GOES and in the hard X-ray flux observed by HXRBS, the flare became detectable at about 19:16 UT (Figure 1). These observations show that there was a gradual onset phase in which the X-ray emission slowly increased until the start of the impulsive phase at about 19:17:30 UT. The impulsive phase produced two major hard X-ray spikes; the first peaked at about 19:18:00 UT, the 
second at about 19:19:15 UT. This double-peaked hard X-ray burst occurred in step with the steep rise of the soft X-ray burst, as is normal. As the hard X-ray emission subsided after its second peak, the soft X-ray flux continued to rise, reached its maximum at about 19:23 UT, then gradually decayed to background level over the next half hour.

The temporal development of the Ov $1371 \AA$ emission in this flare was typical of flare emission from ions formed at transition-region temperatures well below that of the corona $\left(T \ll 10^{6} \mathrm{~K}\right.$; for $\left.\mathrm{Ov}, T \approx 2 \times 10^{5} \mathrm{~K}\right)$; it mimicked the hard $\mathrm{X}$-ray emission, clearly showing the two spikes. A definite quantitative difference is that the two $\mathrm{OV}$ spikes were of nearly equal amplitude, whereas the second hard X-ray spike was about twice as intense as the first (Figure 1b). As we shall see, the OV emission came from the feet of the flare, and the northern foot spread eastward in step with the rise of the second hard X-ray spike. Consequently, a larger fraction of the flare area was within the small raster, and a larger fraction of the total flux of $\mathrm{OV}$ emission from the flare was observed in the second spike than in the first. Hence, for the flare as a whole, the second Ov spike was actually weaker relative to the first spike than indicated by the $\mathrm{OV}$ time profile in Figure 1. So the ratio of the total flux of Ov emission to the total flux of hard $\mathrm{X}$-ray emission in the first spike was at least a factor of 2 greater than in the second spike.

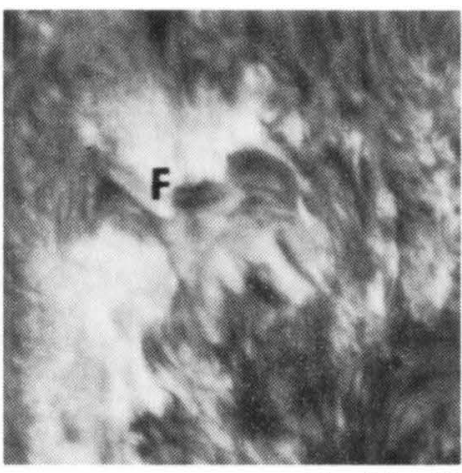

191142

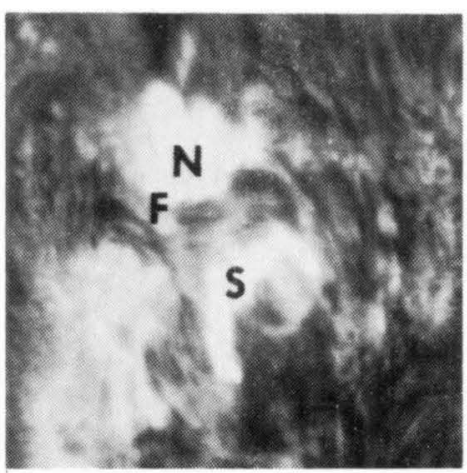

$19 \quad 18 \quad 17$

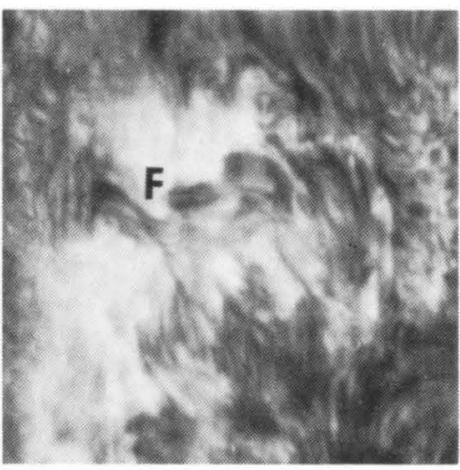

191457

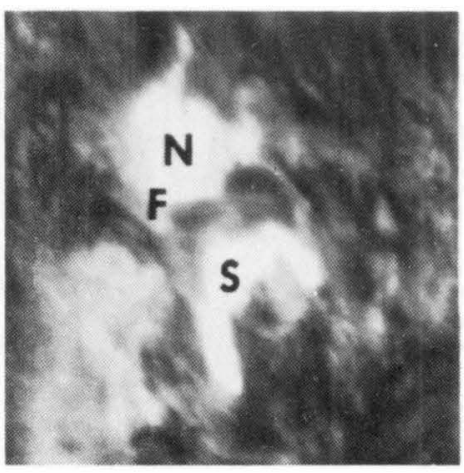

$1919 \quad 11$

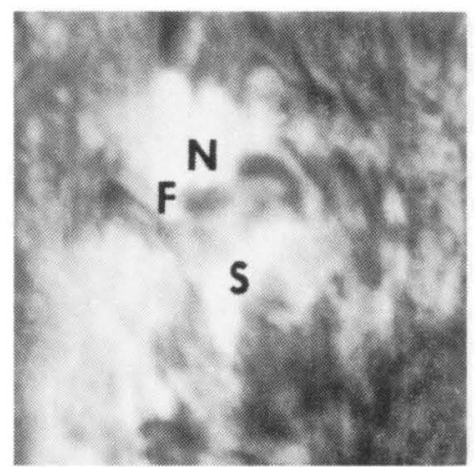

191742

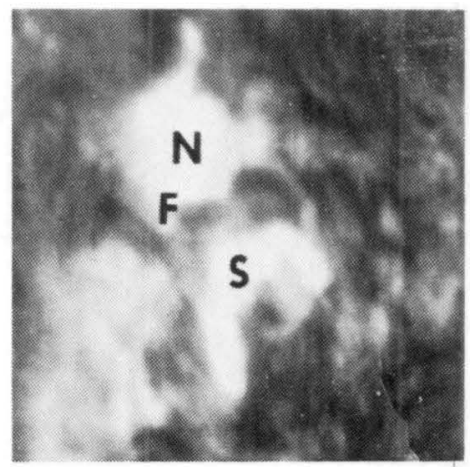

191926

Fig. 4. Structure and development of the flare in $\mathrm{H} \alpha$. The filament marking the magnetic polarity inversion line is labeled F; the two flare ribbons are labeled $\mathrm{N}$ (north) and $\mathrm{S}$ (south). The second frame shows the onset of brightening in progress in both feet. The filament was not appreciably disturbed by the flare. 
In contrast to the $\mathrm{OV}$ emission, the FexXI $1354 \AA$ emission, formed at $T \approx 1 \times 10^{7} \mathrm{~K}$, more nearly mimicked the soft X-ray flux than the hard X-ray flux. Flare UV emission in the vicinity of $1354 \AA$ is a blend of the FeXXI line centered at $1354.05 \AA$ and a narrower Cr line centered at $1354.29 \AA$ (Cheng et al., 1979). Because the OV line is detectable in the absence of flares, but the Fe XXI line is not, the two UVSP exit slits used in these observations were positioned in wavelength by centering one slit on the $\mathrm{O} v$ line prior to the flare. The known offset in wavelength between the slits put the center of the other slit at $1354.09 \AA$ in the FexxI line; it remained in this position through the flare. The width of each slit was 0.3 A. For this exit slit width and position in the Fe XXI line, the spectral observations presented by Cheng et al. (1979) of the decay phase of flares indicate that the $\mathrm{Cr}$ line contributed less than $20 \%$ of the $1354 \AA$ flux observed in our flare after the impulsive phase.

The $1354 \AA$ time profile indicates further that the FexxI emission dominated in the impulsive phase as well (Figure 1). Since the $C_{1}$ line is a fairly weak line formed in the low chromosphere, we expect it to undergo sudden brightening in step with the hard $\mathrm{X}$-ray, $\mathrm{OV}$, and $\mathrm{HeI} \mathrm{D}_{3}$ emissions (Figure 5), and to decay along with these emissions after the impulsive phase. On the other hand, since Fe XXI is formed at $10^{7} \mathrm{~K}$, we expect it to develop in time similarly to the soft X-ray emission. The $1354 \AA$ time profile in Figure 1 does show a small blip in step with the first impulsive spike, and perhaps a hint of the second spike. Except for the blip, the $1354 \AA$ time profile was similar to the 1-8 $\AA$ time profile, although the increase above background was much greater in the $1354 \AA$ emission and its maximum lagged that in 1-8 $\AA$ emission by about $2 \min$ (see Figure 1a). Even if the blip was entirely due to $\mathrm{CI}$ emission, Cr contributed less than half the flux during the blip if the gradual rise though the impulsive phase was due to the growth of FexXI emission expected from the corresponding growth of the 1-8 $\AA$ emission. We therefore conclude that the $1354 \AA$ emission observed by the UVSP in this flare was dominated by FeXXI emission throughout the impulsive phase and after.

The flare onset and spatial development in OV and Fe XXI through the first spike of the impulsive phase is shown in Figure 3. Figure 3 and Figure 2 together show that from onset to at least midway in the impulsive phase, both the OV and Fe XXI emissions were confined to the opposite-polarity foot-points marked by the two $\mathrm{H} \alpha$ ribbons. Since the inner edges of the two ribbons are separated by about $15 \mathrm{arcsec}$ in the impulsive phase (Figure 4), the 10 arc sec resolution of the (contoured) spectroheliograms in Figure 3 results in some blurring between the two ribbons in these images. However, the brightness pattern in the pixel raster verifies that both the $\mathrm{OV}$ and the FeXXI emissions were well confined to the flare feet as late as 19:18:17 (Figure 2c).

During the onset phase, the southern foot was brighter than the northern foot in both $\mathrm{OV}$ and FexxI. Then in the impulsive phase, as shown by the last two pairs of spectroheliograms, which began at 19:17:52 and 19:18:17 UT, the northern foot surpassed the southern foot in brightness. Figures $2 \mathrm{~b}$ and $\mathrm{c}$ show that in $\mathrm{Ov}$ the bipolar pattern with maximum brightness in the northern foot persisted into the decay phase. In contrast, the Fe XXI emission changed from the bipolar pattern to a distribution with a single maximum over the magnetic inversion line between the two flare ribbons. Notice 


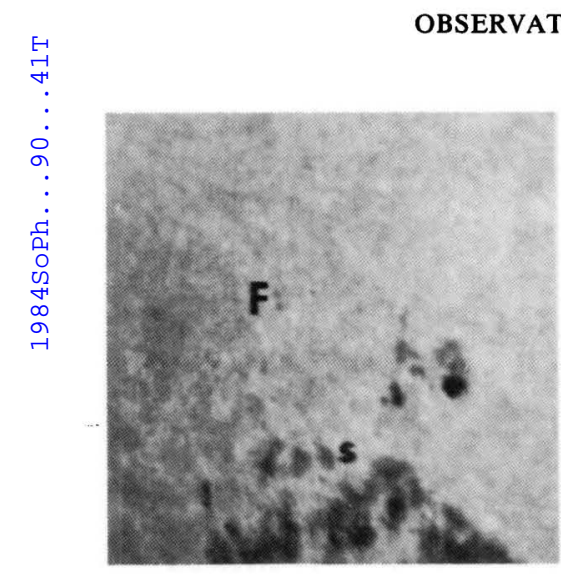

190440
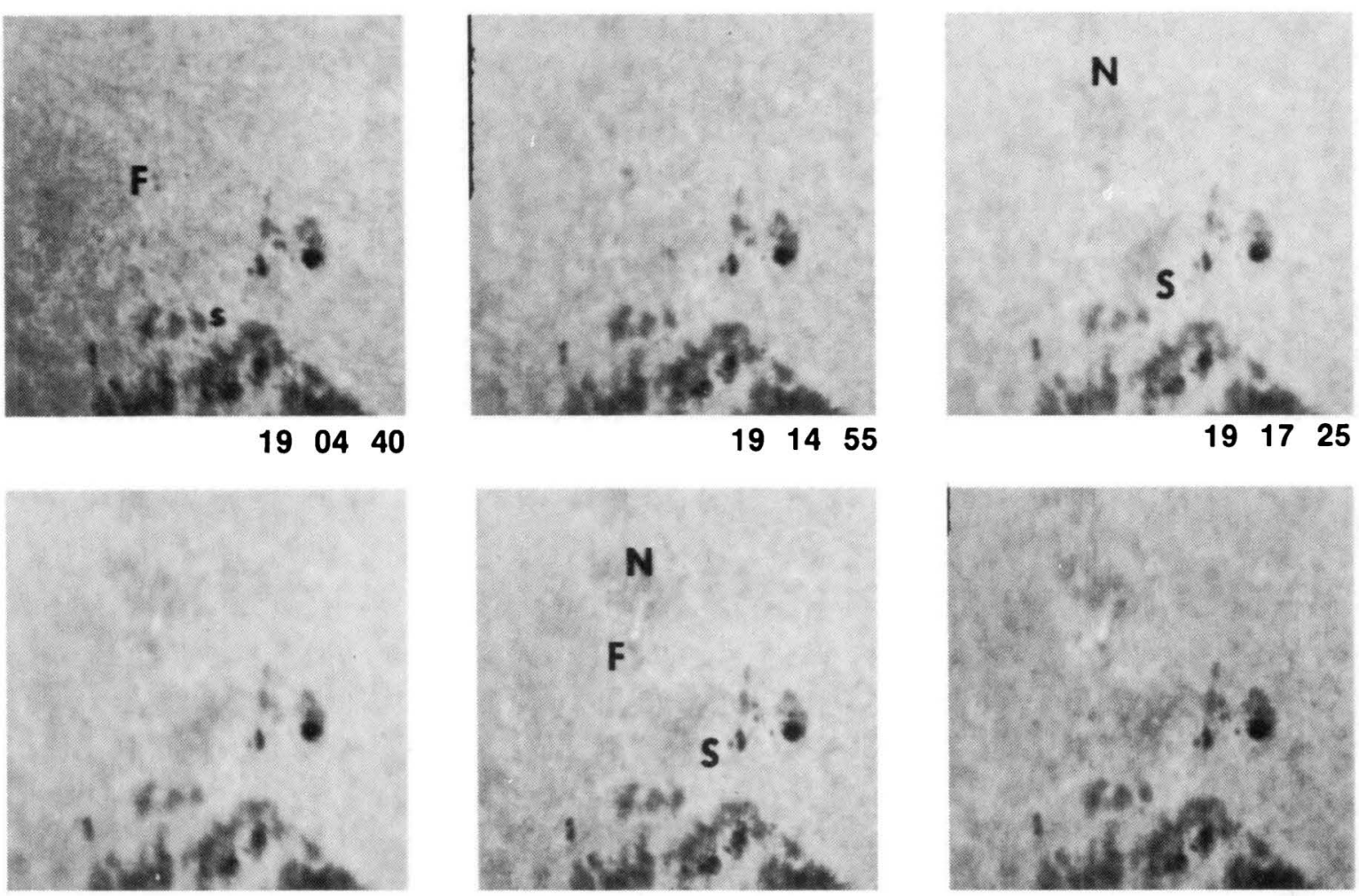

191740

191755

$1918 \quad 19$

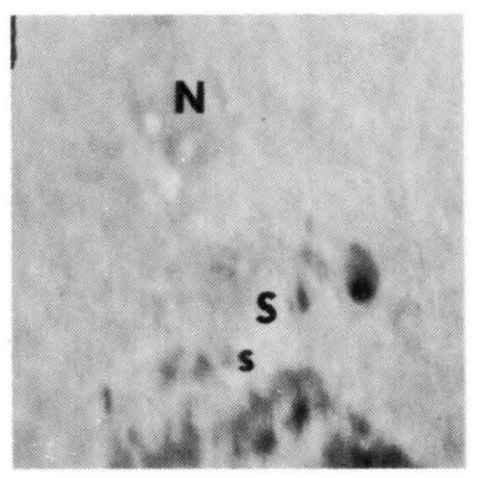

191904
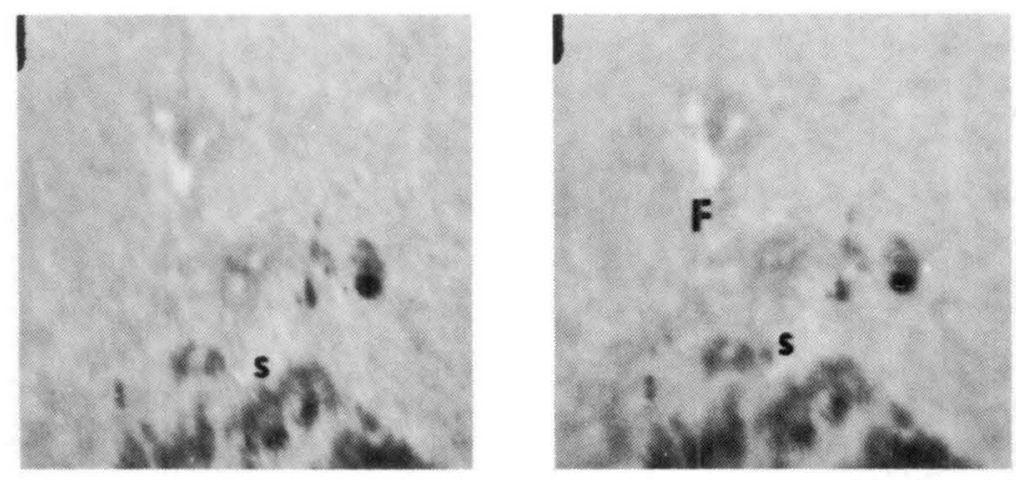

191919

191944

Fig. 5. Structure and development of the flare in $\mathrm{He} I \mathrm{D}_{3}$. The core of the filament and the two flare ribbons are labeled $\mathrm{F}, \mathrm{N}$, and $\mathrm{S}$, as in Figure 4 . The tiny sunspot labeled $s$ is covered by $\mathrm{D}_{3}$ emission in the western end of the southern ribbon at 19:19:19 in the main peak of the impulsive phase. Most of the area of the two ribbons is in absorption and hence dark; in the two impulsive spikes, different kernels in the ribbons brighten in emission (second and third rows). The second frame in the first row shows the onset of the $\mathrm{D}_{3}$ absorption darkening in both feet.

that this brightness maximum is shifted toward the limb to the east with respect to the bright feet in $\mathrm{Ov}$, and that the distribution of brightness is cambered to the east. Since the flare was near the east limb (E64), this indicates that the FeXXI emission at 19: 28 UT was coming mainly from the $T \approx 10^{7} \mathrm{~K}$ thermal X-ray plasma well known to be present at this stage of flares and to be contained in a coronal magnetic arch connecting the opposite-polarity feet of the flare. This confirms that the FexxI line dominated the $1354 \AA$ emission in the postimpulsive thermal phase. 
Figure 4 shows the $H \alpha$ flare from onset through the impulsive phase. As shown by the first panel, a segmented, low-lying filament was in place along the magnetic inversion line prior to the flare. The remaining panels show that this filament was not disturbed by the flare. The $\mathrm{H} \alpha$ movie shows no evidence for eruption or rearrangement of field lines in or between the flare ribbons. Neither is there any evidence in either the magnetogram or the $\mathrm{H} \alpha$ and $\mathrm{D}_{3}$ filtergrams for emerging magnetic flux or any magnetic polarity reversal in the vicinity of the flare other than the main inversion line. All appearances suggest that the flare occurred spontaneously in a magnetic arch rooted in the flare ribbons and looping well above the filament over the inversion line; i.e, this appears to be a fairly simple closed-loop flare.

The $\mathrm{H} \alpha$ movie frame in which the flare brightening was first visible was at $19: 14: 42 \mathrm{UT}$, the frame just before the second panel of Figure 4. In the Ov and Fe XXI spectroheliogram sequences, the flare brightening was first detectable in the filtergram pair which began at 19:14:54 UT, the first filtergram pair in Figure 3. To within the spatial resolution, the emissions in $\mathrm{H} \alpha, \mathrm{Ov}$, and Fe XXI began at the same point in each foot of the flare, to within the temporal resolution the two foot-points turned on together, and the onsets of all three emissions were simultaneous. Of these three lines, the initial brightening above background was strongest in $\mathrm{Ov}$.

The third and fourth panels of Figure 4 are from $\mathrm{H} \alpha$ frames at 19:17:42 and 19:18:17 UT which bracket the peak of the first spike of the impulsive phase. During this spike, both ribbons increased in brightness as the northern ribbon increased slightly in area and the southern ribbon increased significantly in area by growing the horns of a crescent. These new horns were then the brightest parts of the southern ribbon, the brightness of the central part of the crescent having decayed from its maximum at the start of the impulsive phase. These developments in the flare ribbons through the first impulsive spike are also seen in Ov and FeXXI in the last three panels of Figure 3, although the western end of the southern ribbon is outside the field-of-view.

The fifth panel of Figure 4 shows the flare ribbons at the peak of the second impulsive spike. In this spike, the entire area of both ribbons brightened further; the central part of the southern crescent revived to become about as bright as the horns. The area of the northern ribbon expanded slightly eastward and northward while the southern ribbon became somewhat wider and longer. After the last panel of Figure 4 (19:19:26 UT), there was no discerbible further speading of the flare ribbons; they gradually decayed in brightness and area, fading to invisibility over the next half hour.

The development of the flare in $\mathrm{He}$ I $\mathrm{D}_{3}$ from onset through the impulsive phase is shown in Figure 5. The first row of three filtergrams covers the onset phase; the second row spans the first spike of the impulsive phase; the third row is in the second spike. The field-of-view is the same as in Figure 4.

Except in flares, very bright plage, or very dark filaments in $\mathrm{H} \alpha$, the chromosphere is practically transparent in the $\mathrm{HeI} \mathrm{D}_{3}$ line; so outside of such features we see the photosphere in $\mathrm{D}_{3}$ filtergrams. Therefore, $\mathrm{D}_{3}$ filtergrams are well suited so show the location of chromospheric flare ribbons with respect to photospheric magnetic features such as sunspots. Flare ribbons are ususally absorption features in $\mathrm{D}_{3}$; only in the most 
intense $H \alpha$ kernels, usually in the impulsive phase, does $D_{3}$ go into emission. $\mathrm{A} \mathrm{D}_{3}$ filtergram movie thereby shows the foot-points of those magnetic field lines on which the impulsive energy release is concentrated.

In the $\mathrm{D}_{3}$ filtergrams (Figure 5), there was a very small dark feature visible at the location of the darkest part of the middle segment of the $\mathrm{H} \alpha$ filament marking the magnetic inversion line. This feature could possible have been a tiny sunspot; the darker features to the south in Figure 5 were all sunspots. However, because of its proximity to the magnetic inversion line and because it was on the line of sight through the darkest part of the $\mathrm{H} \alpha$ filament, we think it probably was the densest part of the filament seen in $\mathrm{D}_{3}$ absorption. This feature was present before the flare and remained visible and stationary during the flare, as did the $\mathrm{H} \alpha$ filament.

The second panel of Figure 5, at 19:14:55 UT, is from the $\mathrm{D}_{3}$ movie frame in which the initial points of the flare ribbons first became visible; the previous frame was at 19:14: 40 UT. Hence, detectable $\mathrm{D}_{3}$ absorption began simultaneously and cospatially with the flare emission in $\mathrm{H} \alpha, \mathrm{OV}$, and Fe XXI to within the resolution of the observations (see also Feldman et al., 1983). In correspondence with the Ov and Fe XXI emission, the onset in $\mathrm{D}_{3}$ absorption was more visible in the southern foot than in the northern foot of the flare. The third panel of Figure 5, $17 \mathrm{~s}$ before the third panel of Figure 4, shows the growing flare ribbons in absorption at the end of the gradual onset phase.

The fourth panel of Figure 5 shows that a small part of the northern ribbon went into $\mathrm{D}_{3}$ emission in the rise of the first spike of the impulsive phase. The next two panels show that the area of emission spread slightly eastward during the spike. That $D_{3}$ emission was discernible only in the northern ribbon agrees with the northern ribbon becoming brighter than the southern ribbon in $\mathrm{OV}$ and FexxI in the first spike (Figure 3). The last three panels of Figure 5 show that in the second and larger spike of the impulsive energy release, new areas within the northern ribbon went into $D_{3}$ emission, forming a $\mathrm{V}$ pattern with vertex at the eastern extremity of the emission area of the first spike. The brightness of this larger pattern of $\mathrm{D}_{3}$ emission attained its maximum at 19:19:19 UT at the peak of the second impulsive spike. In this peak, faint $\mathrm{D}_{3}$ emission appeared in the central part of the southern ribbon (eighth panel of Figure 5) in synchrony with its renewed brightening in $H \alpha$. There was stronger $D_{3}$ emission at the eastern end of the southern ribbon; this is evident from the temporary disappearance of the small sunspot overlain by that end of the flare ribbon. This sunspot is visible in Figure 5 in all but the eighth panel (at 19:19:19 UT), when the photospheric darkness of the spot was cancelled by the chromospheric flare brightness in $\mathrm{D}_{3}$.

Most of the $\mathrm{D}_{3}$ emission in the second spike was in different areas of the $\mathrm{H} \alpha$ flare ribbons than in the first spike. This indicates that the two spikes of impulsive energy release were centered on different field lines within the flare arch. However, the $\mathrm{D}_{3}$ emission areas in the second spike were mainly within the area of the $\mathrm{H} \alpha$ flare ribbons in the first spike. Therefoer, the second spike of impulsive energy release occurred on field lines which had been involved in or affected by the onset and first impulsive spike of the flare. 
In summary, the main points drawn from our observations of the temporal and morphological development of this flare are the following:

(1) The overall magnetic configuration in which this flare occurred was a fairly simple, closed bipolar arch. However, the very fact that the flare occurred at all and the observed development of the flare ribbons indicate that the arch field was nonpotential and had significant substructure.

(2) The flare apparently occurred spontaneously within the flare arch because: (a) both feet of the flare turned on simultaneously and well away from the inversion line, and (b) there was no evidence of an emerging bipole or other polarity reversal near either initial foot-point.

(3) The FexXI emission came from the feet of the flare well into the impulsive phase, but from over the inversion line between the feet in the decay phase; the Ov emission was concentrated over the feet throughout the flare. Hence, through at least the first minute of the impulsive phase, practically all of the flare-heated plasma at $T \leq 10^{7} \mathrm{~K}$ was confined to the feet of the flare loop.

(4) The two spikes of the impulsive energy release were concentrated in different subsets of field lines within the overall flare loop.

(5) The second spike of impulsive energy release occurred on field lines within the magnetic arch heated in the first spike.

(6) The second impulsive spike produced less OV emission relative to hard X-ray emission than the first spike.

\section{Time History of the UV, X-Ray, and Radio Burst around 19:19 UT}

The relationship between energy bursts at different wavelengths in impulsive flares has been studied by different authors (Crannell et al., 1978, 1982; Kaufmann et al., 1983), and thorough analysis of such relations can help to throw light on the physical mechanisms involved in the flare process itself and in the responses of the solar plasma to the flare disturbance. Since we have excellent time-resolution data in the radio and hard X-ray observations and good time-resolution UV data at the time of the second major impulsive burst at 19:19 UT, we have subjected this data set to further analysis.

Figure 1c shows the second $O V$ and $X$-ray burst at 19:19 UT when we also observed the radio burst at $22 \mathrm{GHz}$; the time profiles of these three impulsive flare emissions are compared over the $120 \mathrm{~s}$ interval starting at 19:18:39 UT. Two questions are of primary interest, viz. the relative timing of the three overall maxima in the UV, X-ray, and radio time profiles, and the character of the fine structure of these curves.

\subsection{TIMING OF BURST MAXIMA}

To compare the relative timing of the maxima in the three emissions, we constructed smoothed curves from a convolution of the data with a low pass Fourier filter using the same cutoff frequency at the 17th harmonic for each time profile. This smoothing operation suppresses all fluctuations with periods less than $7 \mathrm{~s}$ in the observed transients. Figure 6 shows superpositions of data before and after the smoothing. 


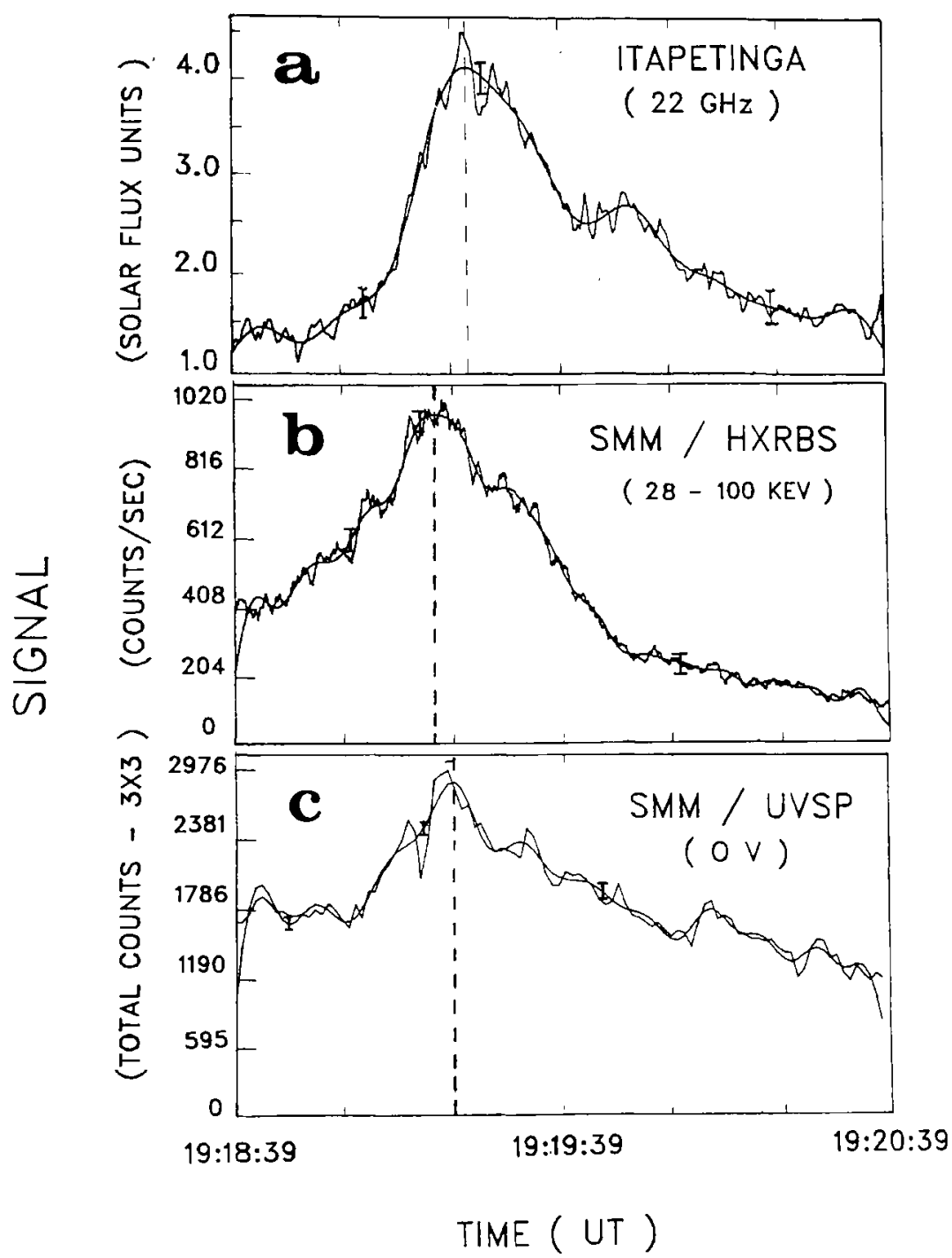

Fig. 6. Comparison of peak times and substructure in the main impulsive burst in (a) the $22 \mathrm{GHz}$ emission, (b) hard X-rays, and (c) Ov, $1371 \AA$. Each smooth profile is the sum of the first 17 Fourier components of the unsmoothed profile; this removes fluctuations with periods less than $7 \mathrm{~s}$. The vertical bars are the $1 \sigma$ amplitudes of expected fluctuations (with periods of order $1 \mathrm{~s}$ ) about the smooth profile, fluctuations expected from solar background noise in the $22 \mathrm{GHz}$ flux and from photon counting statistics in the hard $\mathrm{X}$-ray and $\mathrm{OV}$ bursts. The vertical dashed lines mark the peak times of the smooth profiles. The $22 \mathrm{GHz}$ smooth peak lags the hard X-ray smooth peak by $6 \pm 3 \mathrm{~s}$ (see text). Each of the three profiles shows significant substructure on a time scale of order $10 \mathrm{~s}$, but there is little cross correlation of substructure in any two of the profiles.

The noise in the observations of the impulsive emissions was strong enough that only a few of the largest fluctuations about the smoothed curves in Figure 6 can be confidently attributed to the flare. A vertical bar showing the estimated rms amplitude $( \pm 1 \sigma)$ of the noise is drawn at three points on each of the smoothed time profiles. For the noise in the Ov burst, we took $\sigma=\sqrt{N}$, where $N$ is the number of counts accumulated by the detector during the $1.2 \mathrm{~s}$ interval for one $3 \times 3$ pixel raster. Similary, for the hard X-ray noise, we took $\sigma=\sqrt{N}$, where $N$ is the number of counts per second. The sensitivity of the INPE $22 \mathrm{GHz}$ telescope is $0.03 \mathrm{sfu}$, enough to show that the rms amplitude of 
the short-time $(\approx 1 \mathrm{~s})$ fluctuations sometimes observed from an active region when it is not flaring may typically be about $0.1 \mathrm{sfu}$ (Kaufmann et al., 1982). The bars in Figure 6a therefore represent the solar background fluctuations superimposed on this particular burst and were drawn to span $\sim 0.1 \mathrm{sfu}$. Figure 6 shows that in each of the three emissions the fluctuations in the observed flux about the smoothed curve are of the order expected from the noise. Therefore, while only the largest fluctuations should be considered real substructure of the burst, the substructure of the smoothed curves is true substructure.

We take the peak time of the smoothed profile to be the burst peak time within some uncertainty due to the fluctuations. From the fluctuations actually present within \pm 10 of the peak, we judge the $1 \sigma$ uncertainty in peak time to be about $\pm 2 \mathrm{~s}$ in each of the three emissions.

By this procedure the peak time of the OV burst was at 19:19:19 \pm 02 and the peak time of the hard X-ray burst was at 19:19:15 \pm 02, giving a hardly significant time difference of $4 \pm 3$ s between these two peaks. The lag in the smoothed $O \mathrm{~V}$ peak resulted from the rather large negative fluctuation about $6 \mathrm{~s}$ before the peak; except for this excursion, the profile fo the $\mathrm{OV}$ burst within $\pm 10 \mathrm{~s}$ of the peak was centered on the hard $\mathrm{X}$-ray burst to within about $\pm 1 \mathrm{~s}$ (compare Figures $6 \mathrm{~b}$ and c). Furthermore, the absolute peaks of the two unsmoothed profiles coincided within $1 \mathrm{~s}$. From these observations, we conclude that the $\mathrm{OV}$ and hard X-ray peaks were simultaneous to within $\pm 2 \mathrm{~s}$.

Both the ionization temperature of Ov $\left(2 \times 10^{5} \mathbf{K}\right)$ and our images of the $\mathrm{OV}$ emission indicate that the $\mathrm{OV}$ emission in the impulsive phase came from the feet of the flare. The simultaneity with the hard X-ray peak is consistent with the hypothesis that the impulsive hard X-rays also came from the feet of the flare, where both the hard $\mathrm{X}$-rays and the impulsive heating for the $\mathrm{OV}$ emission were produced by precipitating non-thermal electrons. The observed simultaneity is evidence against the possibility that the peak hard $\mathrm{X}$-ray emission was dominated by thermal bremsstrahlung from a plasma heated to temperatures of order $10^{8} \mathrm{~K}$ near the top of the flare arch. In this case, the peak brightening of the flare feet in $\mathrm{Ov}$ would be expected to lag the impulsive hard $\mathrm{X}$-ray peak by the time for the $10^{8} \mathrm{~K}$ plasma to expand down the legs of the flare loop. In our flare the legs are of order $10^{4} \mathrm{~km}$ long, which gives an expansion time of order $10 \mathrm{~s}$, much too long to satisfy the observed simultaneity. Similar conclusions were drawn by Woodgate et al. (1983) for other flares observed with the SMM UVSP and HXRBS experiments; see also Poland et al. (1982).

In contrast to the unresolvably close match in the times of the OV and hard X-ray peaks, the microwave peak lagged the hard X-ray peak by $6 \pm 3 \mathrm{~s}$. This is confirmed by the lag between the hard X-ray and microwave profiles within $\pm 15 \mathrm{~s}$ of the peak (compare Figures $6 a$ and $b$ ). We checked all available data on the timing accuracy of the hard X-ray and $22 \mathrm{GHz}$ observations and found no reason to doubt this result. (The hard X-ray burst was independently observed at and above $26 \mathrm{keV}$ by the University of California, Berkeley hard X-ray spectral analyzer on the International Sun-Earth Explorer-3. These data verify the absolute timing of the HXRBS observations to within \pm 1 s. The Air Force RSTN Station at Palehua, Hawaii detected the microwave burst 
at $1.415,2.695,4.995$, and $8.800 \mathrm{GHz}$. The emissions at the three highest frequencies peaked within $\pm 1 \mathrm{~s}$ of $19: 19: 21 \mathrm{UT}$, the time of the $22 \mathrm{GHz}$ peak observed at Itapetinga; the $1.415 \mathrm{GHz}$ emission peaked $8 \mathrm{~s}$ later.) Because this result has important implications for the generation of the energetic electrons which produce the impulsive radiation, it is important to obtain simultaneous high-time-resolution hard X-ray and microwave observations of the impulsive phase of other simple loop flares.

Other observations of other flares have indicated that in the impulsive phase the microwaves come from the top of the flare arch while the hard X-rays come from the feet (Hoyng et al., 1981; Marsh and Hurford, 1982). Both the hard X-rays (bremsstrahlung) and the impulsive microwave emission (gyroradiation) come from electrons with energies $\geq 25 \mathrm{keV}$ and velocities $\geq 10^{10} \mathrm{~cm} \mathrm{~s}^{-1}$ (Ramaty et al., 1980). Since the length of our flare arch is $\leq 7 \times 10^{9} \mathrm{~cm}$, electrons accelerated to $\geq 25 \mathrm{keV}$ would travel the length of the arch in less than a second. Therefore, if the electrons for both the hard $\mathrm{X}$-rays and the microwaves were produced at the same time in the same place by the same process, then even if the hard X-rays and microwaves were emitted from different parts of the arch, the lag between the two bursts would be expected to be less than a second. The observation of at least a $3 \mathrm{~s}$ lag between the peaks indicates that the electrons producing the microwave burst were a distinct population, decoupled from the population of electrons producing the hard X-ray burst. From analysis of hard X-ray and microwave spectral data for the impulsive phase of another flare, Marsh et al. (1981) have also found evidence that the hard X-ray and microwave bursts come from different populations of electrons.

\subsection{SubstruCtURE OF THE BURSTS}

In each of the three emissions, the smoothed burst profile shows substructure on a time scale of order $10 \mathrm{~s}$. This is consistent with the overall impulsive phase consisting of elementary flare bursts (de Jager and de Jonge, 1978) which may be built up of many subpulses, quasi-quantized in energy (Kaufmann et al., 1980).

Although the overall peaks of the $\mathrm{O} v$ and hard $\mathrm{X}$-ray bursts are closely simultaneous, there is little correspondence of the substructure in the two emissions. This should perhaps be expected because only part of the northern ribbon was observed in $\mathrm{OV}$ during this burst, while the hard X-ray flux was observed from the entire flare.

In addition to the time lag between the peaks of the hard X-ray and microwave bursts, there is little correspondence of the substructures. The smoothed microwave profile is noticeably smoother than the smoothed hard X-ray profile. The microwave profile shows substructure where the hard X-ray profile is smooth, and vice versa. This lack of correspondence of substructure is further evidence that the electrons producing the microwaves were decoupled from the electrons producing the hard X-rays.

\section{Summary and Discussion}

For our flare, the physical picture which emerges from the observations is sketched in Figure 7 . These cartoons depict the flare arch viewed from the side, so that the underlying 
(a)

\section{PREFLARE: NONPOTENTIAL MAGNETIC ARCH}

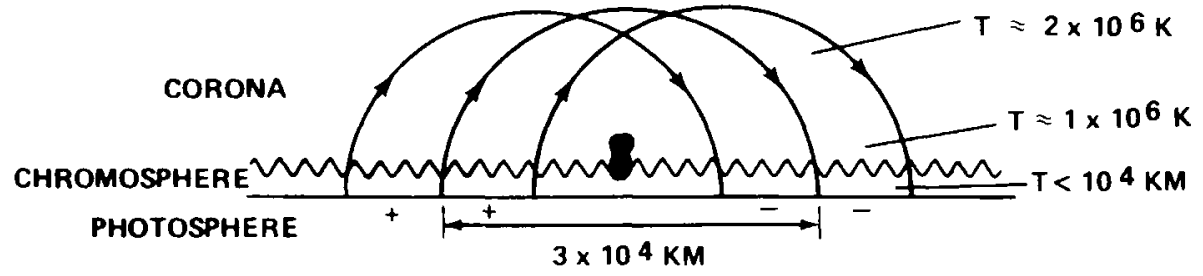

(b)

IMPULSIVE PHASE: CHANGING MAGNETIC SUBSTRUCTURE

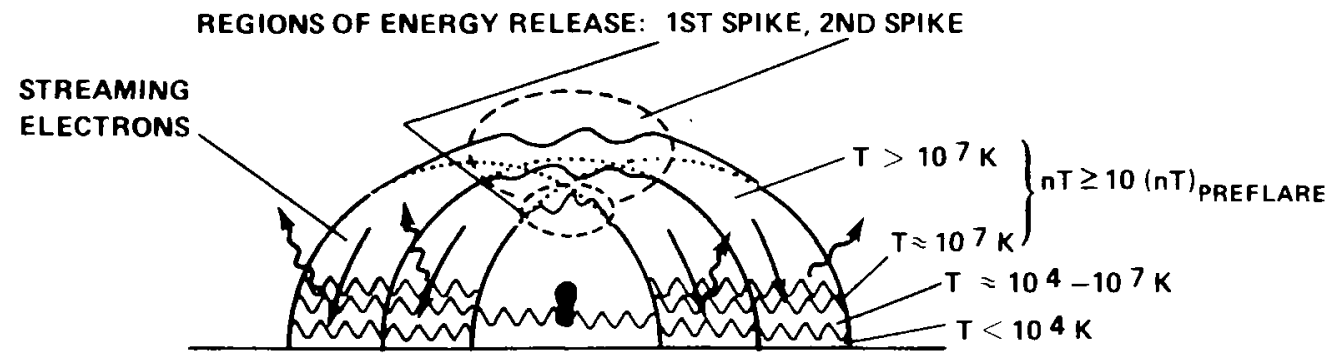

(c)

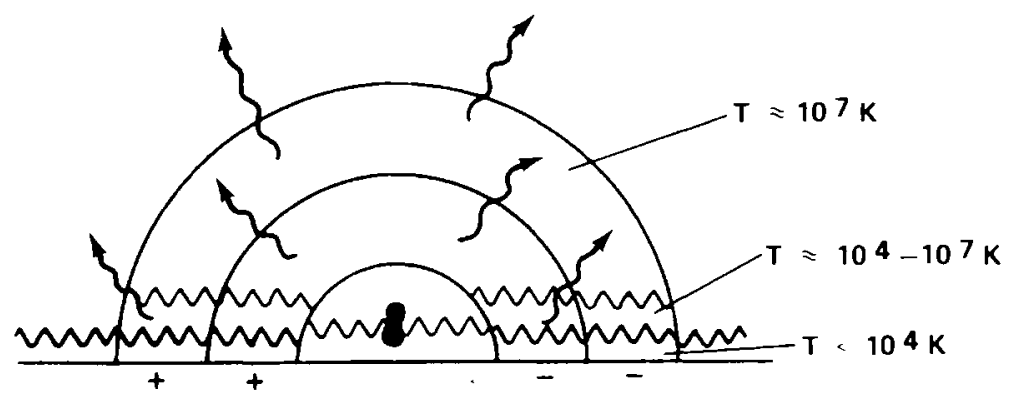

Fig. 7. Schematic physical picture for our flare (a) before, (b) during, and (c) after the impulsive phase. The overall magnetic field configuration was a closed arch before and throughout the flare. The energy release occured in the upper part of the arch as the magnetic substructure rapidly changed from a nonpotential state to a less complex, more nearly potential state. The impulsive energy release immediately increased the temperature (and hence pressure) in the upper part of the arch by at least a factor of 10 , simultaneously with the generation of energetic ( $\geq 10 \mathrm{keV})$ electrons which streamed to the feet. The larger second spike of impulsive energy release occured on field lines on which the corona was preheated in the first spike. The observed lag of the $22 \mathrm{GHz}$ peak behind the hard X-ray peak possibly was produced by the changing magnetic structure. In the impulsive phase, electron precipitation and heat conduction drove chromospheric evaporation; in the decay phase, the coronal flare arch cooled mainly by radiation. 
filament and inversion line are seen end on. The flare arch, flare feet, filament, and height of the chromosphere are drawn with approximately the observed relative positions and proportions; the scale is set by the distance between the feet centroids, about $3 \times 10^{4} \mathrm{~km}$. The shape of the field lines in the flare arch before and after the impulsive phase is taken to be semicircular; this gives a flare arch which passes well above the filament throughout the flare, thus satisfying the observation that the filament was little affected by the flare.

The magnetogram and the high-resolution $\mathrm{D}_{3}$ and $\mathrm{H} \alpha$ filtergrams of the photosphere and chromosphere show no emerging magnetic flux at either foot of the flare. We therefore infer that the flare energy resided in nonpotential magnetic structure in the preflare arch and that the energy release began spontaneously within the arch. Because the two feet turned on and developed synchronously to within a few seconds (judging from the $\mathrm{H} \alpha$ and $\mathrm{D}_{3}$ movies together), we surmise that the energy release occurred mainly in the upper part of the arch. Although, as Figure 7 suggests, there may have been rather strong changes in the magnetic substructure of the arch, there was no evidence of eruption or ejection of field lines and plasma from the flare site; so we surmise that the magnetic field involved in the flare remained closed throughout the flare process. This flare is therefore a good test case for models in which the magnetic field is taken to be a closed loop (Nagai, 1980; Nagai et al., 1983; Emslie, 1981; Doschek et al., 1983).

The observations show several significant features of the flare process in the impulsive phase.

(1) The FeXXI pictures show that for at least the first minute of the impulsive phase, through the first major spike, the $T \approx 1 \times 10^{7} \mathrm{~K}$ plasma was confined to the feet of the flare arch. Prior to the flare, this plasma was presumably at or below the base of the corona and so had a temperature of about $10^{6} \mathrm{~K}$ or less. Hence the pressure in the flare feet immediately increased in the impulsive phase by at least a factor of 10 . If the pressure did not increase the same amount in the rest of the arch above the $10^{7} \mathrm{~K}$ feet, the $10^{7} \mathrm{~K}$ plasma would have expanded up the legs at its sound speed $\left(\approx 500 \mathrm{~km} \mathrm{~s}^{-1}\right)$ and would have filled the arch in about $30 \mathrm{~s}$. Since the FexXI emission area remained well confined to the feet or at least $60 \mathrm{~s}$, we conclude that the pressure immediately increased by at least a factor of 10 all along the length of those field lines on which the impulsive energy release occurred.

It is likely that the density in the $10^{7} \mathrm{~K}$ feet in the impulsive phase was greater than in the upper reaches of the arch. If so, for equal pressure, the temperature in the upper part of the flare arch was greater than $10^{7} \mathrm{~K}$ in the impulsive phase. This is also to be expected because the average temperature of the thermal $\mathrm{X}$-ray plasma in the impulsive phase of flares typically exceeds $10^{7} \mathrm{~K}$ (Moore et al., 1980). Temperatures above $10^{7} \mathrm{~K}$ high in the flare arch would explain why Fe XXI emission was observed only at the feet in the impulsive phase. This would also allow the $10^{7} \mathrm{~K}$ plasma to be continually evaporated into the hotter upper part of the arch. So, there may well have been plasma upflow from the feet in the impulsive phase, upflow in which the plasma was heated above $10^{7} \mathrm{~K}$ (by thermal contact with the hotter plasma) as it welled up out of the $10^{7} \mathrm{~K}$ layer in the feet. This would be a quasi-constant-pressure process in which the density 
in the coronal part of the arch would increase and the temperature decrease, while their product (the pressure) would change relatively little.

(2) The close synchrony of the $O v$ peak with the hard X-ray peak in the second major spike indicates that much of the hard X-ray flux was produced by nonthermal electrons impinging on the flare feet. Thus, it appears that the impulsive energy-release process simultaneously generated very hot $\left(T>10^{7} \mathrm{~K}\right)$ thermal plasma in the body of the arch and energetic ( $>25 \mathrm{keV}$ ) electrons which streamed into the feet. This suggests a hybrid thermal/nonthermal model for the heated/accelerated electrons in the impulsive phase, e.g., as considered by Emslie (1981).

(3) The $D_{3}$ filtergrams show that the two major spikes of impulsive energy release were centered on different field lines within the flare arch; the energy-release site spread to different field lines over the course of the impulsive phase. Therefore, as indicated in Figure 7, the second spike was not a repeated energy release on just the same field lines as in the first spike. In a study of many flares, Zirin (1978) found this to be generally true of flares having multiple impulsive spikes in the energy release.

(4) Although the two spikes of energy release were not centered on the same field lines, the $\mathrm{H} \alpha$ and $\mathrm{D}_{3}$ filtergrams show that the second spike occurred on field lines rooted within the areas of the flare feet that brightened in $\mathrm{H} \alpha$ in the first spike. Hence, the electron acceleration and plasma heating in the second spike took place in coronal loops that were to some degree preheated by the first spike.

It is plausible that the coronal density in the flare arch significantly increased by chromospheric evaporation in the $1 \mathrm{~min}$ interval between the first and second spikes. For example, a subsonic upflow from the $10^{7} \mathrm{~K}$ feet of order $10^{2} \mathrm{~km} \mathrm{~s}^{-1}$ carrying any density $n$ (greater than the initial coronal density in the arch) would increase the density throughout the arch to of order $n$ in a time of order $10^{2} \mathrm{~s}$. A substantial increase in the coronal density before the second spike is consistent with the observation that the ratio of $\mathrm{O} v$ flux to hard $\mathrm{X}$-ray flux was lower in the second spike than in the first by at least by a factor of 2: enhanced coronal density would cause more of the energetic electrons to dissipate in the corona and not reach the feet to produce the heating for the $\mathrm{OV}$ emission. Other evidence for this effect has been found by Strong et al. (1984) in another double impulsive flare.

(5) In the second half of the impulsive phase when there was coverage in both hard $\mathrm{X}$-rays and $22 \mathrm{GHz}$ microwaves, both time profiles show substructure which could be the result of many overlapping subpulses, each having a duration of order $10 \mathrm{~s}$. For a magnetic field strength of order $10^{2} \mathrm{G}$ (which we estimate from the magnetogram to be appropriate for our flare arch) and for electron densities in the range $10^{10}-10^{11} \mathrm{~cm}^{-3}$, the Alfvén speed is of order $10^{3} \mathrm{~km} \mathrm{~s}^{-1}$. So, if the overall length scale of the energyrelease region is of order $10^{4} \mathrm{~km}$, as suggested in Figure 7, then the Alfvén crossing time is of order $10 \mathrm{~s}$. The presence of obvious substructures with time scales of order $10 \mathrm{~s}$ in the impulsive hard X-ray and microwave flux profiles thus suggests that the impulsive energy release was an MHD-driven process.

(6) The $\geq 3 \mathrm{~s} \mathrm{lag}$ between the hard X-ray peak and the microwave peak and the lack of any obvious correspondence in the substructure of the two profiles indicate that the 
microwaves were emitted from a population of electrons that was decoupled from that for the hard X-rays. This may simply mean that the electron populations for the two emissions were accelerated by different mechanisms. Another possibility is that all the energetic electrons were accelerated by a single mechanism, but subsequent interaction with the magnetic field decoupled the microwave electrons from the hard X-ray electrons. For example, as the impulsive energy release progressed in the upper part of the arch, changes in the magnetic structure may have made escape of electrons down the legs progressively more difficult. This could be compatible with the microwaves being emitted mainly from the top of the arch and with the hard X-rays being emitted mainly from the feet, and could result in the hard X-ray flux peaking before the microwave flux. Similar explanations (for time delays between microwave and hard $\mathrm{X}$-ray emissions and between microwave emissions at different frequencies) invoking time-varying magnetic field or acceleration sites in a sequence of differing magnetic structures have been proposed by Brown et al. (1983) and Costa and Kaufmann (1983), and Kaufmann et al. (1982a).

As the impulsive phase ended, the flare feet stopped spreading and began to decay in $\mathrm{H} \alpha$ and $\mathrm{OV}$ brightness. We therefore infer that there was little continued energy release after the impulsive phase. If so, the coronal flare plasma heated in the impulsive phase and contained in the flare arch simply cooled by thermal conduction down the legs of the arch and by direct radiation. In most flares, the conduction drives chromospheric evaporation until the coronal density becomes large enough for direct radiation to dominate the remainder of the cooling; this situation is usually achieved by the time of maximum flux in 1-8 $\AA$ soft X-rays, at which time the density is usually of order $10^{11} \mathrm{~cm}^{-3}$ and the temperature is in the range $1-1.5 \times 10^{7} \mathrm{~K}$ (Moore et al., 1980). The temperature at 1-8 $\AA$ flux maximum in our flare was apparently slightly above $1 \times 10^{7} \mathrm{~K}$ since the Fe XXI emission reached its maximum 2 min later. For temperatures of order $10^{7} \mathrm{~K}$, the radiative cooling time (the temperature $e$-folding time) $\tau_{r}$ is given by $\tau_{r}=1 \times 10^{7} T / n_{e}$ s (Moore et al., 1980). For $n_{e}=10^{11} \mathrm{~cm}^{-3}$ and $T=1 \times 10^{7} \mathrm{~K}$, $\tau_{r}=10^{3} \mathrm{~s}$, which is in reasonable agreement with the observed decay of 1-8 $\AA$ flux in our flare.

\section{Acknowledgements}

We thank Drs R. Shine and W. Henze for sorting out the position of the UVSP exit slit in the FexxI line in our flare. Dr S. Kane kindly and quickly accommodated our request for the UCB/ISEE-3 hard X-ray data. In like manner, Dr S. Kahler and $\mathrm{Mr}$ E. Cliver supplied the Palehua microwave data. Dr D. Rabin prompted us to search for the additional microwave data. RLM was supported by funding from the NASA Solar and Heliospheric Physics Office for research at MSFC on solar magnetic fields and their effects in the solar atmosphere. This research also benefited from RLM's participation in the SMM Workshop on Solar Flares. 


\section{References}

Brown, J. C., MacKinnon, A., Zodi, A. M., and Kaufmann, P.: 1983, Astron. Astrophys., in press.

Cheng, C.-C., Feldman, U., and Doschek, G. A.: 1979, Astrophys. J. 233, 736.

Costa, J. E. R. and Kaufmann, P.: 1983, Astron. Astrophys., in press.

Crannell, C. J., Frost, K. J., Mätzler, C., Ohki, K., and Saba, J. L.: 1978, Astrophys. J. 223, 620.

Crannell, C. J., Karpen, J. T., and Thomas, R. J.: 1982, Astrophys. J. 253, 975.

de Jager, C. and de Jonge, G.: 1978, Solar Phys. 58, 127.

Doschek, G. A., Cheng, C.-C., Oran, E. S., Boris, J. P., and Mariska, J. T.: 1983, Astrophys. J. 265, 1103.

Emslie, A. G.: 1981, Astrophys. J. 245, 711.

Feldman, U., Liggett, M., and Zirin, H.: 1983, Astrophys. J., in press.

Hagyard, M. J., Cumings, N. P., West, E. A., and Smith, J. E.: 1982, Solar Phys. 80, 33.

Hoyng, P., Duijveman, A., Machado, M. E., Rust, D. M., Švestka, Z., Boelee, A., de Jager, C., Frost, K. J., LaFleur, H., Simnett, G. M., Van Beek, H. F., and Woodgate, B. E.: 1981, Astrophys. J. 246, L155.

Kaufmann, P., Strauss, F. M., Opher, R., and Laporte, C.: 1980, Astron. Astrophys. 87, 58.

Kaufmann, P., Costa, J. E. R., and Strauss, F. M.: 1982a, Solar Phys. 81, 159.

Kaufmann, P., Strauss, F. M., Schaal, R. E., and Laporte, C.: 1982b, Solar Phys. 78, 389.

Kaufmann, P., Strauss, F. M., Costa, J. E. R., Dennis, B. P., Kiplinger, A., Frost, K. J., and Orwig, L. E.: 1983, Solar Phys. 84, 311.

Marsh, K. A. and Hurford, G. J.: 1982, Ann. Rev. Astron. Astrophys. 20, 497.

Marsh, K. A., Hurford, G. J., Zirin, H., Dulk, G. A., Dennis, B. R., Frost, K. J., and Orwig, L. E.: 1981, Astrophys. J. 251, 797.

Moore, R., McKenzie, D. L., Švestka, Z., Widing, K. G., Antiochos, S. K., Dere, K. P., Dodson-Prince, H. W., Hiei, E., Krall, K. R., Krieger, A. S., Mason, H. E., Petrasso, R. D., Pneuman, G. W., Silk, J. K., Vorpahl, J. A., and Withbroe, G. L.: 1980, in P. A. Sturrock (ed.), Solar Flares, Colorado Associated University Press, p. 341.

Nagai, F.: 1980, Solar Phys. 68, 351.

Nagai, F., Wu, S. T., and Tandberg-Hanssen, E.: 1983, Solar Phys. 84, 271.

Orwig, L. E., Frost, K. J., and Dennis, B. R.: 1980, Solar Phys. 65, 25.

Poland, A. I., Machado, M. E., Wolfson, C. J., Frost, K. J., Woodgate, B. E., Shine, R. A., Kenny, P. J., Cheng, C. C., Tandberg-Hanssen, E. A., Bruner, E. C., and Henze, W.: 1982, Solar Phys. 78, 201.

Ramaty, R., Colgate, S. A., Dulk, G. A., Hoyng, P., Knight, J. W., Lin, R. P., Melrose, D. B., Orrall, F., Paizis, C., Shapiro, P. R., Smith, D. F., and Van Hollebeke, M.: 1980, in P. A. Sturrock (ed.), Solar Flares, Colorado Associated University Press, p. 117.

Strong, K. T., Benz, A. O., Dennis, B. R., Leibacher, J. W., Mewe, R., Poland, A., Schrijver, J., Simnett, G., Smith, J. B., Jr., and Sylwester, J.: 1984, Solar Phys., in press.

Woodgate, B. E., Tandberg-Hanssen, E. A., Bruner, E. C., Beckers, J. M., Brandt, J. C., Henze, W., Hyder, C. L., Kalet, M. W., Kenny, P. J., Knox, E. D., Michalitsianos, A. G., Rehse, R., Shine, R. A., and Tinsley, H. D.: 1980, Solar Phys. 65, 73.

Woodgate, B. E., Shine, R. A., Poland, A., and Orwig, L. E.: 1983, Astrophys. J. 265, 530.

Zirin, H.: 1978, Solar Phys. 58, 95. 